\title{
Dynamical Phase Transition from Nonequilibrium Dynamics of Dark Solitons
}

\author{
Minyong Guo, ${ }^{1,2,3,{ }^{*}}$ Esko Keski-Vakkuri, ${ }^{4, \dagger}$ Hong Liu, ${ }^{5, *}$ Yu Tian, ${ }^{6,7,8}$ and Hongbao Zhang ${ }^{1,8, \|}$ \\ ${ }^{1}$ Department of Physics, Beijing Normal University, Beijing 100875, China \\ ${ }^{2}$ Perimeter Institute for Theoretical Physics, Waterloo, Ontario N2L 2Y5, Canada \\ ${ }^{3}$ Center for High Energy Physics, Peking University, Beijing 100871, China \\ ${ }^{4}$ Helsinki Institute of Physics and Department of Physics, University of Helsinki, Helsinki FIN 00014, Finland \\ ${ }^{5}$ Center for Theoretical Physics, Massachusetts Institute of Technology, Cambridge, Massachusetts 02139, USA \\ ${ }^{6}$ School of Physics, University of Chinese Academy of Sciences, Beijing 100049, China \\ ${ }^{7}$ Institute of Theoretical Physics, Chinese Academy of Sciences, Beijing 100190, China \\ ${ }^{8}$ Theoretische Natuurkunde, Vrije Universiteit Brussel, and The International Solvay Institutes, \\ Pleinlaan 2, B-1050 Brussels, Belgium
}

(Received 13 May 2019; published 21 January 2020)

\begin{abstract}
By holographic duality, we identify a novel dynamical phase transition which results from the temperature dependence of nonequilibrium dynamics of dark solitons in a superfluid. For a nonequilibrium superfluid system with an initial density of dark solitons, there exists a critical temperature $T_{d}$, above which the system relaxes to equilibrium by producing sound waves, while below which it goes through an intermediate phase with a finite density of vortex-antivortex pairs. In particular, as $T_{d}$ is approached from below, the density of vortex pairs scales as $\left(T_{d}-T\right)^{\gamma}$ with the critical exponent $\gamma=1 / 2$.
\end{abstract}

DOI: 10.1103/PhysRevLett.124.031601

Introduction.-Nonequilibrium superfluid states obtained from external driving or phase-density imprinting often contain a large number of dark solitons [1-7], which are regions of low densities in a superfluid. The subsequent evolution of such a system and its relaxation to equilibrium, where the involved dark solitons play a vital role, are of great interest. Instabilities of dark solitons in various superfluids were studied extensively in the literature at the theoretical level (see, e.g., Refs. [8-12]), mainly based on the Gross-Pitaevskii equation or Bogoliubov-de Gennes equation [13-15], which is suitable only for zero temperature. To address the finite temperature dissipative effect, the above equations or the variants become less reliable. For instance, the dissipative Gross-Pitaevskii equation [16], where a dissipative parameter is added "by hand," is rather crude and requires significant modeling.

Compared to this, holographic duality, which equates certain strongly correlated systems of quantum matter without gravity to classical gravitational systems in a curved spacetime with one additional spatial dimension, provides us with a complementary theoretical framework, in which the superfluid at finite temperature is dual to a hairy black hole in the bulk and the dissipation mechanism is naturally built in the bulk in terms of excitations absorbed

Published by the American Physical Society under the terms of the Creative Commons Attribution 4.0 International license. Further distribution of this work must maintain attribution to the author(s) and the published article's title, journal citation, and DOI. Funded by SCOAP. by the hairy black hole. Such a framework provides a firstprinciples description of finite temperature dissipative effect in the sense that once the "microscopic" theory is fixed, all aspects of the superfluid phase are determined without the phenomenological modeling involved.

In this Letter, we employ such a holographic tool to perform a systematic study of temperature dependence of the dynamics and decay mechanisms of dark solitons, where we find there exists a critical temperature $T_{d}$ such that for $T>T_{d}$ the dark soliton decays dominantly by selfaccelerating uniformly and then turning into sound waves but for $T<T_{d}$ by fragmenting into vortex pairs or filaments a process called snake instability. We argue that this pattern persists with a finite density of dark solitons, which results in a novel dynamical phase transition for the relaxation process of superfluids at $T_{d}$. For $T>T_{d}$, the solitons decay directly into sound waves which subsequently relax to equilibrium, while for $T<T_{d}$, an intermediate phase with a finite density of vortices emerges; see Fig. 1. The intermediate phase is unstable but could in principle be long-lived enough for interesting dynamics including turbulence to happen. The density $n_{v}$ of vortices, which may be considered as the "order parameter" of the intermediate phase, is proportional to the density of original dark solitons and increases as the temperature is decreased. In particular, near $T_{d}$, it has critical behavior:

$$
n_{v} \propto\left(T_{d}-T\right)^{\gamma}, \quad \gamma=\frac{1}{2}
$$




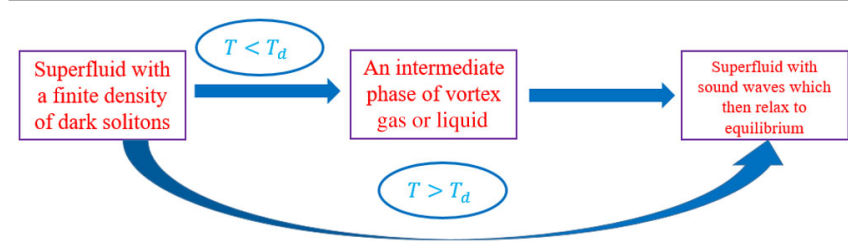

FIG. 1. For $T<T_{d}$ there is an intermediate phase of vortex gas or liquid.

We also present a general analytic argument for the "critical exponent" $\frac{1}{2}$ in Eq. (1).

Holographic setup and dark soliton solutions.-Let us start from the holographic superfluid in $2+1$ dimensions, which can be described by an Abelian-Higgs model in a $(3+1)$-dimensional anti-de Sitter (AdS) black hole spacetime $[17,18]$. The Lagrangian can be written as

$$
\mathcal{L}=-\frac{1}{4} F_{a b} F^{a b}-|(\nabla-i A) \Psi|^{2}-m^{2}|\Psi|^{2},
$$

with the background spacetime metric

$$
d s^{2}=\frac{L^{2}}{z^{2}}\left[-f(z) d t^{2}-2 d t d z+d x^{2}+d y^{2}\right]
$$

where $L$ is the curvature radius of $\operatorname{AdS}$ and $f(z)=$ $1-\left[\left(z / z_{h}\right)\right]^{3}$, with $z=z_{h}$ the location of an event horizon and $z=0$ the AdS boundary. The black hole spacetime Eq. (3) has a Hawking temperature $T=\left(3 / 4 \pi z_{h}\right)$, which is identified with the temperature of the dual boundary system. In Eq. (2) the $U(1)$ gauge field $A_{a}$ is dual to a conserved particle current $J^{a}$ for a $U(1)$ global symmetry in the boundary system, and the complex scalar field $\Psi$ is dual to a boundary order parameter $\psi$ charged under the $U(1)$ symmetry. The system is in a superfluid phase below some critical temperature $T_{c}$, when $\Psi$ develops a normalizable profile in the bulk spacetime which corresponds to the boundary condensate $\psi$ developing a nonzero expectation value $[17,18]$. We will ignore the backreaction of $A_{a}$ and $\Psi$ to the background black hole geometry, an approximation which works well when the temperature is not too low [19]. We are interested mostly in the regime $T / T_{c} \gtrsim 0.3$, where the approximation is sufficient.

For simplicity we will take the mass square of $\Psi$ to be $m^{2}=-\left(2 / L^{2}\right)$. In this case, there are two possible boundary conditions for $\Psi$, leading to two different types of superfluids which we denote, respectively, as $\psi_{ \pm}$. For definiteness, below we focus solely on the $\psi_{+}$superfluid. We emphasize that once the parameters and boundary conditions of the gravity description (2) are fixed, the system is fully specified at all scales, including the full set of properties of the superfluid phase such as the critical temperature, temperature dependence of the order parameter, and so on.
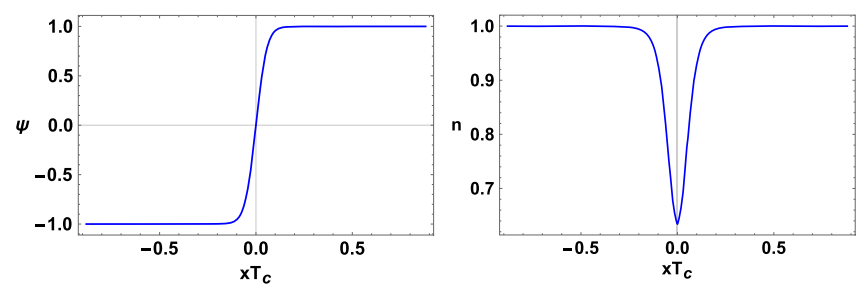

FIG. 2. The profiles for the condensate and particle density of a dark soliton at $\left(T / T_{c}\right)=0.48$ (normalized to 1 at infinity).

A static dark soliton is a defect in a superfluid. For definiteness, we shall take it to be translation invariant along the $y$ direction and centered at $x=0$. The corresponding condensate $\psi_{s}(x)$, up to the overall phase factor, has the following characteristic properties,

$$
\begin{gathered}
\bar{\psi}_{s}(x)=\psi_{s}(x), \quad \psi_{s}(-x)=-\psi_{s}(x), \\
\psi_{s}(x \rightarrow+\infty) \rightarrow \psi_{h} \quad \text { exponentially fast, }
\end{gathered}
$$

with $\psi_{h}$ the condensate in the homogeneous phase. Near the center, the particle density also gets depleted. As demonstrated in Fig. 2, such dark soliton solutions $\psi_{s}$ can be found by solving equations of motion following from Eq. (2) with appropriate boundary conditions. For details, see Supplemental Material, where we have presented all the numerics involved in this Letter [20].

Linear instability analysis and switch of dominant decay channel.-Now consider small perturbations around a dark soliton solution. Since the dark soliton configuration is independent of $t$ and $y$, in the linearized equations of motion, all the bulk perturbed fields can be written in terms of Fourier modes $e^{-i \omega t+i q y}$ multiplied by the corresponding unknown functions of $x$ and $z$, which can be solved together with $\omega$ for each $q$ as a generalized eigenvalue problem. Because of the parity symmetry $y \rightarrow-y, q$ and $-q$ have identical behavior, so we will restrict to $q \geq 0$. Furthermore, due to the parity property of the dark soliton with respect to $x \rightarrow-x$ indicated in Eq. (4), one finds that the even and odd eigenmodes decouple. We denote $\omega_{e}(q), \omega_{o}(q)$, respectively, as the corresponding eigenvalues, which have a discrete spectrum and are all complex. As a result, at the linear level we have

$$
\psi(t, x)=\psi_{s}(x)+\epsilon f_{\omega, q}(x) e^{-i \omega t+i q y},
$$

with $\epsilon$ a small parameter. Note that an $\omega$ eigenvalue with a positive imaginary part leads to exponential time growth in Eq. (6) and thus corresponds to an unstable mode.

At a given temperature $T$, one finds that there exists a $q_{c}(T)$ such that there is exactly one unstable mode for each $q \in\left[0, q_{c}(T)\right)$ and the mode is even in $x$. The unstable mode turns out to be pure imaginary $\omega_{e}^{(0)}(q)=i \lambda_{q}$ with 


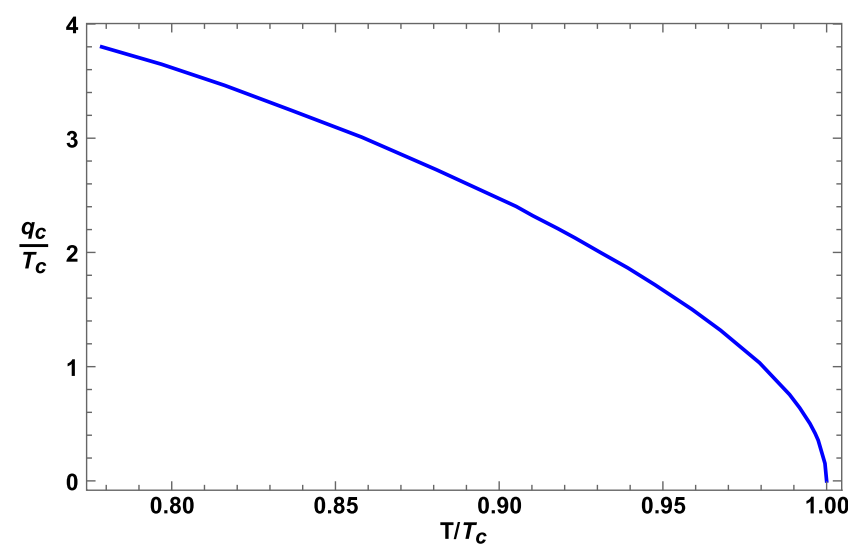

FIG. 3. The plot of $q_{c}(T)$ as a function of temperature.

$\lambda_{q}>0$, which leads to $e^{-i \omega_{e}^{(0)}(q) t}=e^{\lambda_{q} t}$. We will denote the eigenfunction $f_{\omega, q}(x)$ for the unstable mode as $f_{q}^{(0)}(x)$.

As illustrated in Fig. 3, the upper value $q_{c}(T)$ for the unstable mode decreases as one increases the temperature, and $q_{c}(T) \rightarrow 0$ as $T \rightarrow T_{c}$. This is reasonable because $q_{c}(T)$ is proportional to the healing length, which is divergent at $T_{c}$. More explicitly, we find that as $T \rightarrow T_{c}$,

$$
q_{c}(T) \approx 2.704 T_{c}\left(1-T / T_{c}\right)^{1 / 2} .
$$

We will now show that (i) the unstable mode at $q=0$ corresponds to self-acceleration, (ii) the unstable mode at $q \neq 0$ corresponds to snake instability, and (iii) when a soliton decays via snake instability, there is a vortexantivortex pair to be produced for each wavelength. For example, consider the system in a finite periodic box with length $R_{y}$ along the $y$ direction. The allowed $q$ 's are thus of the form $q=\left(2 \pi N / R_{y}\right)$, with $N$ an integer. A snake instability with $q=\left(2 \pi N / R_{y}\right)$ will then create $N$ pairs of vortices. To see (i), let us note that the center $x_{c}$ of the dark soliton can be identified as being located at the minimum of the condensate; i.e., $\left.\left(\partial|\psi|^{2} / \partial x\right)\right|_{x_{c}}=0$. Now consider Eq. (6) with $f_{\omega, q}$ given by $f_{q=0}^{(0)}(x)$, i.e., $\psi=\psi_{s}(x)+\epsilon f_{q=0}^{(0)}(x) e^{\lambda_{0} t}$, which leads to

$$
|\psi|^{2}=\psi_{s}^{2}+2 \epsilon \psi_{s}(x) \operatorname{Re} f_{q=0}^{(0)}(x) e^{\lambda_{0} t} .
$$

It can readily be seen from the above equation that

$x_{c}=-\epsilon \frac{\eta_{0}}{\xi} e^{\lambda_{0} t}$, with $\eta_{0}=\operatorname{Re} f_{q=0}^{(0)}(0), \quad \xi=\partial_{x} \psi_{s}(0)$.

We thus see that the dark soliton accelerates exponentially.

Now let us consider Eq. (6) for a general unstable $q$, with Eq. (8) becoming

$$
|\psi|^{2}=\psi_{s}^{2}+2 \epsilon \psi_{s}(x) \operatorname{Re}\left(f_{q}^{(0)}(x) e^{i q y}\right) e^{\lambda_{q} t} .
$$

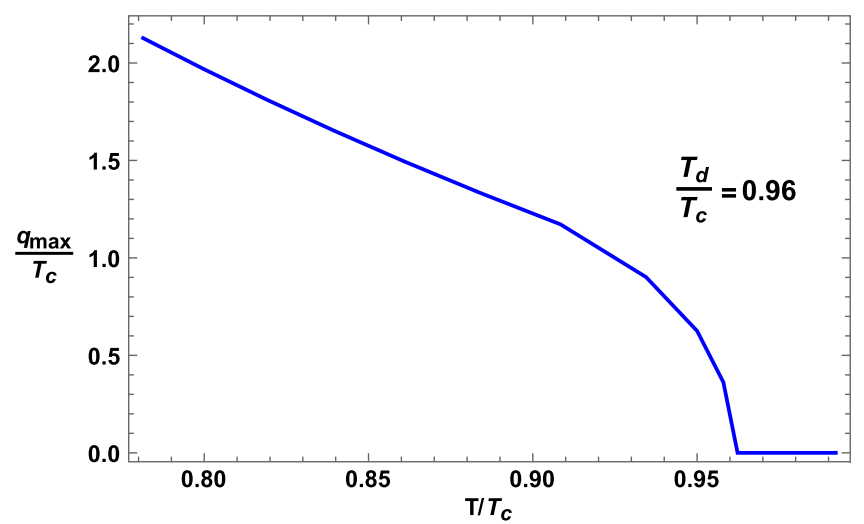

FIG. 4. The plot of $q_{\max }(T)$ as a function of temperature.

We then have

$x_{c}(t, y)=-\epsilon \frac{\eta_{q}}{\xi} \cos (q y+\theta) e^{\lambda_{q} t}, \quad \eta_{q}=\left|f_{q}^{(0)}(0)\right|$,

and $\theta=\arg f_{q}^{(0)}(0)$. From Eq. (11), $x_{c}$ depends on $y$ sinusoidally, leading to a snake instability. In particular, for each wavelength $(2 \pi / q)$ there are two points of zero velocity with opposite circulations around them, corresponding to the locations of a vortex and an antivortex to be created. This thus demonstrates (ii) and (iii) above.

Among all the unstable modes $q<q_{c}(T)$, the one with the maximal $\lambda_{q}$ grows fastest and is thus expected to be the dominant decay channel of a soliton. In Fig. 4 we plot the value of $q_{\max }(T)$ for which the maximal value of $\lambda_{q}$ occurs as a function of temperature. We see that $q_{\max }(T)$ decreases with temperature monotonically until a value $T_{d}<T_{c}$, after which $q_{\max }$ becomes identically zero. Furthermore, as $T \rightarrow T_{d}$ from below, we find the following "critical behavior":

$$
q_{\max } \propto\left(T_{d}-T\right)^{\gamma},
$$

with the exponent $\gamma$ numerically around $\frac{1}{2}$. Actually, there is a simple Ginzburg-Landau-type argument which shows that $\gamma$ should be given by $\frac{1}{2}$ generically. Because of the aforementioned parity symmetry, one can consider expanding $\lambda_{q}(T)$ in small $q$ as

$$
\lambda_{q}(T)=\lambda_{0}(T)-\frac{1}{2} a(T) q^{2}-\frac{1}{4} b(T) q^{4}+\cdots .
$$

Since for $T \approx T_{d}, q_{\max }$ is close to zero, and the above expansion should suffice for determining the behavior of $q_{\max }$ near $T_{d}$. For $T \gtrsim T_{d}, q_{\max }$ is zero; thus we should have $a(T)>0$, while for $T \lesssim T_{d}$, we should generically have $a(T)<0, b\left(T_{d}\right)>0$ in order to have $q_{\max } \neq 0$. We thus conclude that near $T=T_{d}$ we can expand $a(T)$ as

$$
a(T)=a_{0}\left(T-T_{d}\right)+\cdots, \quad a_{0}>0 .
$$

It then follows that 


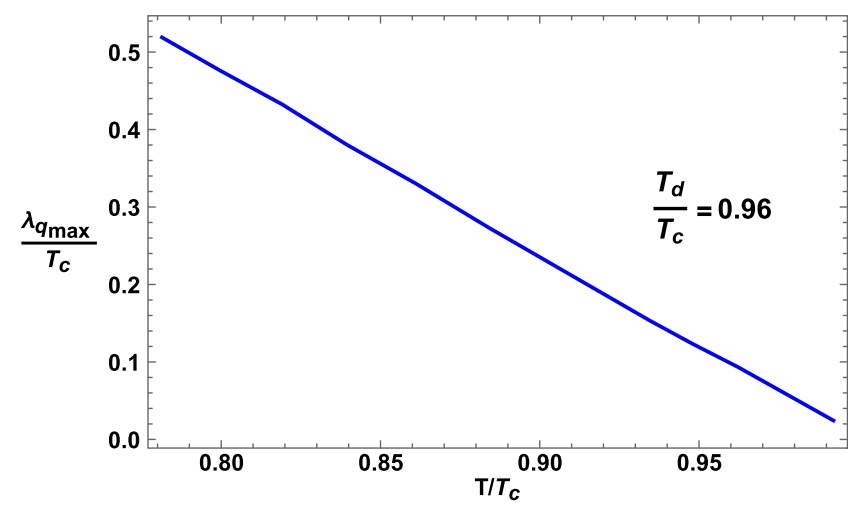

FIG. 5. The plot of $\lambda_{q_{\max }}$ as a function of temperature.

$q_{\max }=\sqrt{\frac{a_{0}\left(T_{d}-T\right)}{b_{0}}}, \quad T \lesssim T_{d}, \quad b_{0}=b\left(T_{d}\right)>0$.

From our earlier discussion of self-acceleration and snake instability, we thus conclude that there is a sharp transition at $T_{d}$ in how dark solitons decay: for $T_{c}>T>T_{d}$, dark solitons decay by self-acceleration, while for $T<T_{d}$, they decay by snake instability. In particular, in the snake instability regime, the number of vortex and antivortex pairs created by the decay of a dark soliton, which is proportional to $q_{\max }$, decreases as we increase the temperature.

The value $\lambda_{q_{\max }}$ characterizes the growth rate of instabilities and thus its inverse may be considered as giving the timescale for the "lifetime" of a dark soliton. In Fig. 5 we plot the temperature dependence of $\lambda_{q_{\max }}$. It is interesting to note the higher the temperature is, the slower the instabilities grow and thus the longer lifetime a soliton has.

Full nonlinear simulation and dynamical phase transition.-To confirm our expectation that the decay of a dark soliton is controlled by the $q_{\max }$ mode with the largest $\lambda_{q}$, we now perform the full nonlinear evolution by considering the dynamics of a single dark soliton initiated by the following two types of perturbations: (i) a singlewavelength perturbation of the form $\delta \Psi \propto z^{2} e^{i q y}$ with $q \in\left[0, q_{c}(T)\right)$ and (ii) a general perturbation of the form $\delta \Psi \propto z^{2} \sum_{q} e^{i \alpha_{q}} e^{i q y}$ with random phases $\alpha_{q}$ 's, which turn out to give a generic decay pattern of a single dark soliton. For (i), with $q=0$, one finds that different parts of the dark soliton accelerate uniformly. During its acceleration, the dark soliton also broadens, and eventually dissolves into sound waves. For $q \neq 0$, the acceleration pattern for different parts of the soliton shows sinusoidal behavior with wavelength $(2 \pi / q)$, consistent with the prediction of Eq. (11). In particular, the vortex-antivortex formation as well as their numbers are precisely as predicted below Eq. (11); see Figs. 6(a) and 6(b). For a general perturbation (ii), the resulting evolutions are shown in Figs. 6(c) and 6(d). We indeed find that at a temperature above $T_{d}$, the soliton accelerates without any vortex formation [Fig. 6(c)]. At a temperature below $T_{d}$, the evolution is dominated by snake

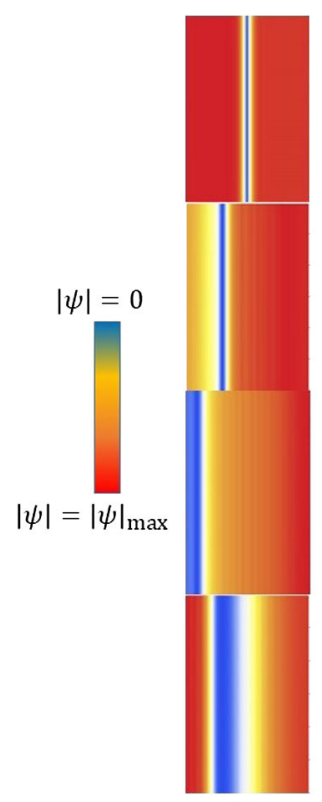

(a)

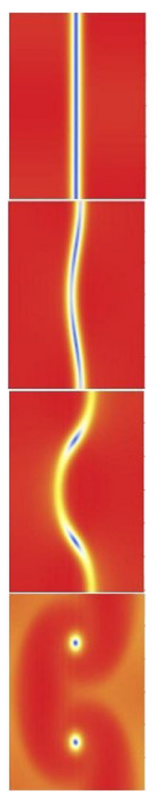

(b)

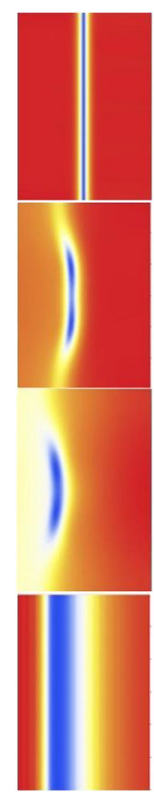

(c)

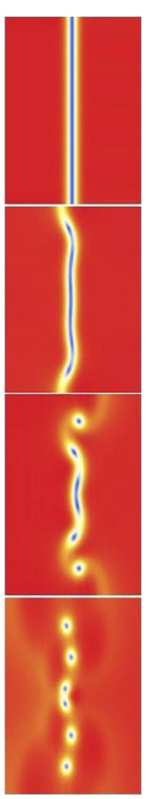

(d)
FIG. 6. The density plots of the time evolution of a dark soliton under various initial perturbations. Time increases from the top to the bottom in each panel. The perturbations for (a) and (b) have the form $\delta \Psi \propto z^{2} e^{i q y}$ with (a) $q=0$ and (b) $q=\left(2 \pi / R_{y}\right)$ at $\left(T / T_{c}\right)=0.78$. The perturbations for (c) and (d) are of the form $\delta \Psi \propto z^{2} \sum_{q} e^{i \alpha_{q}} e^{i q y}$ with (c) at $\left(T / T_{c}\right)=0.99$ and (d) at $\left(T / T_{c}\right)=0.78$.

instability, evidenced by formation of vortex pairs. Furthermore, the number of vortex pairs is consistent with linear analysis. For instance, at $\left(T / T_{c}\right)=0.78$, the linear analysis tells us that the mode $q=\left(2 \pi N / R_{y}\right)$ with $N=3$ is the most unstable mode. We find the nonlinear evolution indeed produces 3 vortex pairs [Fig. 6(d)].

On physical ground, we expect our results regarding the stability of a single soliton should apply to a finite density of solitons as far as the density is not too high, i.e., as far as the average distances between solitons are larger than the healing length of a soliton. That is to say, for such a nonequilibrium superfluid state, there is a nonequilibrium dynamical phase transition at $T_{d}$ in how the system relaxes back to equilibrium. For $T>T_{d}$, the solitons directly decay to sound waves which subsequently equilibrate. For $T<T_{d}$, there exists an intermediate phase with an initial density $n_{v}$ of vortex-antivortex pairs, which is proportional to $q_{\max }$. The lower the temperature is, the larger $q_{\max }$ and $n_{v}$. Furthermore, from Eq. (12), as $T_{d}$ is approached from below, $n_{v} \propto q_{\max }$ has the critical behavior given by Eq. (1).

Note that $\left(T_{d} / T_{c}\right)=0.96$, so the occurrence of such a dynamical phase transition lies well in the applicability regime of the probe limit. Consequently, we do not expect a qualitative change about the above picture even if the backreaction effect is taken into account.

Discussion.-To summarize, by holographic studying the decay of dark solitons, we identified a novel dynamical 
phase transition which results from the temperature dependence of nonequilibrium dynamics of solitons. The phase transition involves the appearance of an intermediate (unstable) phase with the order parameter given by the density of vortex pairs and a critical exponent $\gamma=\frac{1}{2}$. This vortex gas-liquid phase could have important implications for understanding nonequilibrium dynamics of a superfluid; for example, it could be long-lived enough to exhibit vortex and wave turbulent states.

As detailed in the companion paper [29], such a dynamical phase transition is found to occur also in the $\psi_{\text {- }}$ superfluid, strongly suggesting that it is a universal phenomenon predicted by holography. It would be extremely interesting to search for this novel dynamical phase transition in experiments and to measure the critical temperature $T_{d}$ as well as the critical exponent $\gamma$. A natural place to look for such a phase transition is ultracold atomic gases. The transition should already be detectable in the usual experimental setup of a conventional harmonic potential. Recent experimental advances in the realization of boxlike optical traps [30] can make the comparisons with our theoretical results even more direct.

M. G. is partially supported by National Natural Science Foundation of China (NSFC) with Grants No. 11675015, No. 11775022, No. 11875095, and No. 11947210, as well as by China Scholarship Council (CSC). He is also funded by China National Postdoctoral Innovation Program 2019M660278. He also thanks the Perimeter Institute Visiting Graduate Fellows program for support. Research at Perimeter Institute is supported by the Government of Canada through the Department of Innovation, Science and Economic Development and by the Province of Ontario through the Ministry of Research, Innovation and Science. E. K-V. is partially supported by the Academy of Finland Grant No. 1297472, and by a grant from the Vilho, Yrjö and Kalle Väisälä Foundation. H. L. is partially supported by the Office of High Energy Physics of the U.S. Department of Energy under grant Contract No. DE-SC0012567. Y. T. is partially supported by NSFC with Grant No. 11975235 and he is also supported by the Strategic Priority Research Program of the Chinese Academy of Sciences with Grant No. XDB23030000. H.Z. is supported in part by Fonds Wetenschappelijk Onderzoek (FWO)-Vlaanderen through the project G006918N, and by the Vrije Universiteit Brussel through the Strategic Research Program "High-Energy Physics." He is also an individual FWO fellow supported by $12 \mathrm{G} 3518 \mathrm{~N}$.

*minyongguo@mail.bnu.edu.cn

†esko.keski-vakkuri@helsinki.fi

"hong_liu@mit.edu

§ytian@ucas.ac.cn

"hzhang@vub.ac.be
[1] S. Burger, K. Bongs, S. Dettmer, W. Ertmer, K. Sengstock, A. Sanpera, G. V. Shlyapnikov, and M. Lewenstein, Phys. Rev. Lett. 83, 5198 (1999).

[2] J. Denschlag et al., Science 287, 97 (2000).

[3] C. Becker, S. Stellmer, P. Soltan-Panahi, S. Dörscher, M. Baumert, E.-M. Richter, J. Kronjäger, K. Bongs, and K. Sengstock, Nat. Phys. 4, 496 (2008).

[4] S. Stellmer, C. Becker, P. Soltan-Panahi, E.-M. Richter, S. Dörscher, M. Baumert, J. Kronjäger, K. Bongs, and K. Sengstock, Phys. Rev. Lett. 101, 120406 (2008).

[5] A. Weller, J. P. Ronzheimer, C. Gross, J. Esteve, M. K. Oberthaler, D. J. Frantzeskakis, G. Theocharis, and P. G. Kevrekidis, Phys. Rev. Lett. 101, 130401 (2008).

[6] D. J. Frantzeskakis, J. Phys. A 43, 213001 (2010).

[7] M. J. H. Ku, B. Mukherjee, T. Yefsah, and M. W. Zwierlein, Phys. Rev. Lett. 116, 045304 (2016).

[8] A. E. Muryshev, H. B. van Linden van den Heuvell, and G. V. Shlyapnikov, Phys. Rev. A 60, R2665(R) (1999).

[9] Th. Busch and J. R. Anglin, Phys. Rev. Lett. 84, 2298 (2000).

[10] Th. Busch and J. R. Anglin, Phys. Rev. Lett. 87, 010401 (2001).

[11] D. M. Gangardt and A. Kamenev, Phys. Rev. Lett. 104, 190402 (2010).

[12] A. Cetoli, J. Brand, R. G. Scott, F. Dalfovo, and L. P. Pitaevskii, Phys. Rev. A 88, 043639 (2013).

[13] E. P. Gross, Il Nuovo Cimento 20, 454 (1961).

[14] L. P. Pitaevskii, Sov. Phys. JETP 13, 451 (1961).

[15] P. G. De Gennes, Superconductivity of Metals and Alloys (Benjamin, New York, 1966).

[16] L. P. Pitaevskii, Zh. Eksp. Teor. Fiz. 35, 408 (1958) [Sov. Phys. JETP 35, 282 (1959)].

[17] S. S. Gubser, Phys. Rev. D 78, 065034 (2008).

[18] S. A. Hartnoll, C. P. Herzog, and G. T. Horowitz, Phys. Rev. Lett. 101, 031601 (2008).

[19] S. A. Hartnoll, C. P. Herzog, and G. T. Horowitz, J. High Energy Phys. 12 (2008) 015.

[20] See Supplemental Material at http://link.aps.org/ supplemental/10.1103/PhysRevLett.124.031601 for the details about our numerical computation, which includes Refs. [21-28].

[21] W. J. Li, Y. Tian, and H. Zhang, J. High Energy Phys. 07 (2013) 030.

[22] S. Q. Lan, Y. Tian, and H. Zhang, J. High Energy Phys. 07 (2016) 092.

[23] V. Keranen, E. Keski-Vakkuri, S. Nowling, and K. P. Yogendran, Phys. Rev. D 80, 121901(R) (2009).

[24] V. Keranen, E. Keski-Vakkuri, S. Nowling, and K. P. Yogendran, Phys. Rev. D 81, 126011 (2010).

[25] A. Adams, P. M. Chesler, and H. Liu, Science 341, 368 (2013).

[26] C. Ewerz, T. Gasenzer, M. Karl, and A. Samberg, J. High Energy Phys. 05 (2015) 070.

[27] Y. Du, C. Niu, Y. Tian, and H. Zhang, J. High Energy Phys. 12 (2015) 018.

[28] P. M. Chesler, A. M. Garcia-Garcia, and H. Liu, Phys. Rev. X 5, 021015 (2015).

[29] M. Guo, E. Keski-Vakkuri, H. Liu, Y. Tian, and H. Zhang (to be published).

[30] A. L. Gaunt, T. F. Schmidutz, I. Gotlibovych, R. P. Smith, and Z. Hadzibabic, Phys. Rev. Lett. 110, 200406 (2013). 